\title{
Determinación in vitro de la Actividad Antimicótica del Aceite de Romero (Rosmarinus officinalis) sobre Microsporum canis
}

\author{
In vitro Determination of Antifungal Activity of Rosemary Oil (Rosmarinus \\ officinalis) on Microsporum canis
}

\author{
Sandra Dentone ${ }^{1}$, Siever Morales Cauti ${ }^{2}$
}

\section{Resumen}

\begin{abstract}
El objetivo del presente estudio fue determinar la actividad antimicótica in vitro del aceite de romero (Rosmarinus Officinalis) sobre Microsporum canis. Se aisló una cepa de $M$. canis a partir de muestras clínicas. El aceite de romero se obtuvo por el método de arrastre a vapor con una concentración del $100 \%$ de pureza. Mediante el método de difusión en pocillo, se enfrentó la cepa de $M$. canis a ocho concentraciones del aceite de romero $(250,500,1000,2000,5000,10000,50000$ y 100000 ppm). Los resultados indicaron una adecuada sensibilidad de $M$. canis a partir de la concentración de 50000 ppm.
\end{abstract}

Palabras clave: aceite de romero, Microsporum canis

\section{Abstract}

The aim of this study was to determine the antifungal in vitro activity of rosemary oil (Rosmarinus officinalis) against Microsporum canis. A strain of M. canis was isolated from clinical samples. The rosemary oil was obtained by the method of steam distillation with a purity of $100 \%$. By the well diffusion method the strain of $M$. canis was faced against eight concentrations of rosemary oil (250, 500, 1000, 2000, 5000, 10000,50000 and $100000 \mathrm{ppm})$. The results showed an adequate sensitivity of $M$. canis at a concentration of 50000 ppm onwards.

Key words: rosemary oil, Microsporum canis

\footnotetext{
${ }^{1}$ Laboratorio de Microbiología, Carrera de Medicina Veterinaria y Zootecnia, Universidad Cientifica del Sur, Lima, Perú

${ }^{2}$ Laboratorio de Microbiología y Parasitología Veterinaria, Facultad de Medicina Veterinaria, Universidad Nacional Mayor de San Marcos, Lima, Perú

${ }^{3}$ E-mail: sieverm@hotmail.com
}

Recibido: 3 de mayo de 2016

Aceptado para publicación: 2 de setiembre de 2016 


\section{INTRODUCCIÓN}

La creciente resistencia a antimicrobianos y la menor disponibilidad económica para adquirir fármacos ha propiciado la búsqueda de nuevos compuestos como alternativa terapéutica contra las micosis (Domingo y Lopez-Brea, 2003; Abad et al., 2007; Guerra, 2011). Principalmente, se buscan productos de origen natural, que sean seguros, no tóxicos, eficaces y de bajo costo; por ello, los productos naturales como el aceite de romero son una excelente opción a investigar.

En el Perú, Ruiz y Roque (2009) evaluaron los efectos medicinales de cuatro plantas del nororiente del país (Cassia reticulata, Ilex guayusa Loes, Piper lineatum y Terminalia catappa). Algunos extractos presentaron actividad antimicrobiana frente a Staphylococcus aureus, S. epidermidis, Escherichia coli, Candida albicans y Microsporum canis. Asimismo, estudios en plantas medicinales utilizadas en Brasil y Colombia demostraron que diversos extractos redujeron el crecimiento de hongos, incluyendo M. canis (Lizcano y Vergara, 2009; Rodríguez y Kozusny-Andreani, 2010). Estos resultados sustentan el estudio de la determinación del efecto inhibitorio del aceite de romero frente a $M$. canis aislado de cuadros clínicos.

Una de las plantas más utilizadas de manera casera en la población peruana es el romero (Rosmarinus officinalis), debido a sus múltiples propiedades medicinales (Purca, 2013). El romero pertenece a la familia Lamiaceae, planta arbustiva con tallos prismáticos, cuyas hojas finas, estrechas, agudas y pequeñas contienen aceites esenciales con diversos principios activos (Ruiz et al., 1975; López, 2008; Diaz et al., 2011).

El aceite esencial de romero presenta propiedades antimicrobiales contra una variedad de agentes patógenos, posiblemente debido al alto contenido de 1,8-cineol (Estrada, 2010; Diaz et al., 2011; Teixeira, 2012). Una de las patologías más comunes en la clínica de animales menores es la dermatofitosis, producida generalmente por los dermatofitos Microsporum, Trichopytum y Epidermophytum, donde el Microsporum canis es el dermatofito más común en perros y gatos. La infección por $M$. canis se presenta a menudo en forma asintomática. Se considera una antropozoonosis asociada a pequeños animales que afecta piel, pelo y uñas (Scott et al., 2002).

El método más fiable para el diagnóstico de una dermatofitosis por $M$. canis es mediante el cultivo, donde se pueden observar las características macroscópicas (forma y color de las colonias) y microscópicas (presencia de macroconidias y microconidias) (Gueaguere, 2007). Además, existen pruebas rápidas como la lámpara de Wood, así como el examen directo de pelos, escamas o raspado de uñas donde se puede observar las artrosporas o hifas sobre el material parasitado. Asimismo, la biopsia de piel es un método no necesario, aunque es efectivo (Birchard y Sherding, 2002).

El tratamiento para la dermatofitosis canina depende de la extensión de las lesiones. Así pues, el tratamiento tópico es utilizado en lesiones aisladas, utilizándose productos antimicóticos como ketoconazol, miconazol, clotrimazol o terbinafina en forma de pomadas, soluciones y cremas, en tanto que para las lesiones generalizadas se puede usar champús antimicóticos y tratamiento sistémico (Escobedo, 2011). El fármaco de elección para las infecciones sistémicas es la griseofulvina, pero también se utiliza el ketoconazol e itraconazol, aunque suelen producir efectos adversos como vómitos, diarrea, y anorexia, además de ser hepatotóxicos (Maddison et al., 2004; Plumb, 2010). Además, los tratamientos suelen ser efectivos después de varios meses (Cervantes, 2003). Por ello, el presente estudio evalúa el uso de alternativas naturales, como los aceites esenciales del romero, para el control de este tipo de infecciones. 


\section{Materiales y Métodos}

El estudio se llevó a cabo en el Laboratorio de Microbiología de la Universidad Científica del Sur, distrito de Villa el Salvador, departamento de Lima.

El tamaño de la muestra $(\mathrm{n}=80)$ se determinó según la fórmula de determinación de tamaño de muestra para diferencia de proporciones (Mateu y Casal, 2003), con nivel de confianza $\left(Z_{\mathrm{a}}=2.58\right)$, poder de prueba $\left(\mathrm{Z}_{\mathrm{a}}=1.282\right)$, proporción control $\left(\mathrm{P}_{1}=0.1\right) \mathrm{y}$ proporción tratamiento $\left(\mathrm{P}_{2}=0.75\right)$.

El aceite de romero fue extraído por el método de destilación por arrastre a vapor (Cerpa, 2007). La cepa de M. canis fue aislada de un paciente clínicamente afectado.

Se realizaron 80 evaluaciones, empleando ocho concentraciones del aceite esencial $(250,500,1000,2000,5000,10000,50000 \mathrm{y}$ 100000 ppm), además del control positivo de ketoconazol al $1 \%$ y el control negativo, con ocho repeticiones cada uno.

La siembra de $M$. canis se realizó en 32 placas petri con agar Sabouraud, mediante la técnica de arrastre con el hisopo sobre toda la superficie del agar en tres direcciones. Luego, se perforó el agar en 4 puntos con un sacabocado de $11 \mathrm{~mm}$, los que se inocularon hasta formar una superficie cóncava con el aceite esencial en sus diferentes concentraciones (dilutor: DimetildisulfoxidoDMSO). En las primeras 8 placas se colocaron las concentraciones de aceite esencial de romero de 250, 500, 1000 y $2000 \mathrm{ppm}$, en las siguientes 8 placas se colocaron las concentraciones de 5000, 10000,50000 y $100000 \mathrm{ppm}$ ). El control positivo (ketoconalzol al 1\%) se colocó en 8 placas y el control negativo (agua destilada estéril) en las restantes 8 placas.

Las placas se incubaron a temperatura ambiente por 7 días. Los resultados se registraron según ell tamaño del halo (diámetro de inhibición del aceite de romero sobre la cepa de M. canis). Se compararon los halos de inhibición obtenidos con el reporte de la Asociación Argentina de Fitomedicina sobre los resultados de la concentración mínima inhibitoria (Flores et al., 2004). Se fijaron tres categorías: sensible (S), intermedia (I) y resistente (R), según se describe en el Cuadro 1. Para los efectos del estudio se midió el diámetro (= radio x 2).

Cuadro 1. Interpretación de la actividad antimicótica sobre Microsporum canis según halo de inhibición

\begin{tabular}{ll}
\hline $\begin{array}{l}\text { Actividad } \\
\text { inhibitoria }\end{array}$ & Radio del halo \\
\hline Sensible & Mayor a $9 \mathrm{~mm}$ \\
Intermedia & De 6 a $9 \mathrm{~mm}$ \\
Resistente & Menor de $6 \mathrm{~mm}$ \\
\hline
\end{tabular}

Fuente: Flores et al., 2004

\section{Resultados}

Los resultados obtenidos de la actividad antimicótica para el presente estudio se evidencian a partir de concentraciones mayores de 5000 ppm del aceite de romero, con un promedio de $10.5 \mathrm{~mm}$ de diámetro de inhibición para 10000 ppm del aceite evidenciando una actividad inhibitoria frente al Microsporum canis aislado de cuadros clínicos en perros con dermatitis micótica (Cuadro 2).

De acuerdo a la interpretación, el Microsporum canis se muestra sensible a la actividad inhibitoria del aceite de romero a una concentración de 50000 ppm con un porcentaje de $75 \%$ de la cepa en sus repeticiones (Cuadro 3). La validación de la técnica se desarrolló a través de los controles positivos (ketoconazol 1\%) en pastilla y crema, con resultados de actividad antimicótica ma- 
Cuadro 2. Actividad antimicótica de varias concentraciones de aceite de romero ${ }^{1}$ sobre el Microsporum canis, según el halo de inhibición

\begin{tabular}{ccccccc}
\hline \multirow{2}{*}{ Repetición } & \multicolumn{6}{c}{ Diámetro del halo de inhibición $(\mathrm{mm})$} \\
\cline { 2 - 7 } & $\begin{array}{c}5000 \\
\mathrm{ppm}\end{array}$ & $\begin{array}{c}10,000 \\
\mathrm{ppm}\end{array}$ & $\begin{array}{c}50,000 \\
\mathrm{ppm}\end{array}$ & $\begin{array}{c}100,000 \\
\mathrm{ppm}\end{array}$ & $\begin{array}{c}\text { Control } \\
\text { positivo }\end{array}$ & $\begin{array}{c}\text { Control } \\
\text { negativo }\end{array}$ \\
\hline 1 & 0 & 10 & 12 & 18 & $>50$ & 0 \\
2 & 0 & 8 & 26 & 32 & $>50$ & 0 \\
3 & 0 & 12 & 28 & 30 & $>50$ & 0 \\
4 & 0 & 8 & 18 & 20 & $>50$ & 0 \\
5 & 0 & 12 & 22 & 40 & $>50$ & 0 \\
6 & 0 & 14 & 28 & 30 & $>50$ & 0 \\
7 & 0 & 10 & 30 & 34 & $>50$ & 0 \\
8 & 0 & 10 & 28 & 36 & $>50$ & 0 \\
\hline Promedio & 0 & 10.5 & 24 & 30 & $>50$ & 0 \\
\hline & &
\end{tabular}

Cuadro 3. Interpretación del halo de inhibición sobre $M$. canis de tres concentraciones de ac eite esencial de romero

\begin{tabular}{cccc}
\hline Concentración $(\mathrm{ppm})$ & Resistente & Intermedio & Sensible \\
\hline 10,000 & $62.5 \%(5 / 8)$ & $37.5 \%(3 / 8)$ & $0 \%(0 / 8)$ \\
50,000 & $0 \%(0 / 8)$ & $25 \%(2 / 8)$ & $75 \%(6 / 8)$ \\
100,000 & $0 \%(0 / 8)$ & $12.5 \%(1 / 8)$ & $87.5 \%(7 / 8)$ \\
\hline
\end{tabular}

yor de 50 mm de inhibición, y el control negativo (suero fisiológico) con actividad de $0 \mathrm{~mm}$ de inhibición (Cuadro 3).

\section{Discusión}

M. canis demostró ser sensible in vitro a las concentraciones de $50000(75 \%)$ y 100000 ppm (87.5\%) de aceite de romero. Este aceite presenta, además, un amplio espectro de acción antibacteriana, tanto sobre bacterias Gram positivas como Gram negativas, aunque a diferentes concentraciones (Castaño et al., 2010), posiblemente debido a la menor complejidad de los componentes de la pared celular de las bacterias, como son peptidoglucanos y fosfolípidos, y a través de los diversos componentes de los aceites esenciales (Estrada, 2010).

Las propiedades biológicas del aceite esencial de romero se atribuyen, generalmente, a sus componentes mayoritarios, como los 
compuestos terpénicos, tales como los sesquiterpenos y sesquiterpenlactonas (Abad et al., 2007; Estrada, 2010); sin embargo, en ocasiones estos componentes actúan en sinergia con otros componentes minoritarios, como los heterósidos, flavónicos, polifenoles, flavonoides y alcaloides, favoreciendo las propiedades biológicas (Bakkali et al., 2008).

Los extractos de romero han demostrado actividad antibacteriana frente a Staphylococcus aureus, Candida albicans y Pseudomonas aeruginosa (Estrada, 2010), Leishmania braziliensis (Arevalo et $a l ., 2009)$. Sin embargo, en la mayoría de reportes se demuestra su actividad contra hongos filamentosos y levaduras (Tabanca et al., 2006).

El control utilizado para la evaluación del aceite esencial de romero fue ketoconazol al $1 \%$, el cual es el fármaco de elección para el tratamiento de las dermatofitosis, tanto sistémico como localmente. Actúa inhibiendo la síntesis de ergosterol, provocando una actividad enzimática relacionada con la membrana, lo que conduce a un aumento de la permeabilidad e inhibición del crecimiento celular y su replicación (Sumano y Ocampo, 1997). En este estudio, los halos de inhibición del fármaco fueron mayores de $50 \mathrm{~mm}$, lo que valida los resultados obtenidos.

\section{Conclusión}

Microsporum canis es sensible in vitro al aceite esencial de romero a una concentración de 50000 ppm.

\section{Literatura Citada}

1. Abad M, Ansuategui M, Bermejo P. 2007. Active antifungal substances from natural sources. ARKIVOC 7: 116-145.

2. Arévalo Y, Robledo $S$, Muñoz D, Granados-Falla D, Cuca L, Delgado G. 2009. Evaluación in vitro de la acti- vidad de aceites esenciales de plantas colombianas sobre Leishmania braziliensis. Rev Colomb Cienc Quím Farm 38: 131-141.

3. Bakkali F, Averbeck S, Averbeck D, Idaomar M. 2008. Biological effects of essential oils - A review. Food Chem Toxicol 46: 446-475. doi: 10.1016/ j.fct.2007.09.106

4. Birchard S, Sherding R. 2002. Manual clínico de procedimientos en pequeñas especies. $2^{\circ}$ ed. México: McGrawHill. 1941 p.

5. Castaño H, Ciro G, Zapata J, Jiménez S. 2010. Actividad bactericida del extracto etanólico y del aceite esencial de hojas de Rosmarinus officinalis L sobre algunas bacterias de interés alimentario. VITAE 17: 149-154.

6. Cerpa MG 2007. Hidrodestilación de aceites esenciales: modelado y caracterización. Tesis de Doctorado. Valladolid, España: Universidad de Valladolid. $304 \mathrm{p}$.

7. Cervantes $R A$. 2003. Ringworm infection in $\operatorname{dog} s$ and cats. In: Carmichael L (ed). Recent advances in canine infectious diseases. Ithaca: IVIS. [Internet]. Disponible en: http:// ww w . ivis . org/advances/ infect_dis_carmichael/cervantes/ivis.pdf

8. Díaz $P$, Cabrera $M$, Alem $D$, Larrañaga $P$, Ferreira F. Dalla $M$. 2011. Antifungal activity of medicinal plant extracts against phytopathogenic fungus Alternaria spp. Chilean J Agric Res 71:231-239.

9. Domingo D, López-Brea M. 2003. Plantas con acción antimicrobiana. Rev Esp Quimioterap 16: 385-393.

10. Escobedo J. 2011. Dermatofitosis, diagnóstico y tratamiento. [Internet]. Disponible en: http://dermatologiaveterinaria puebla.blogspot.com/2011/04/ dermatofitosis.html

11. Estrada S. 2010. Determinación de la actividad antibacteriana in vitro de los extractos de romero (Rosmarinus officinalis) y tomillo (Tymus vulgaris). Tesis de Bioquímico Farmacéutico. Riobamba, Ecuador: Escuela Superior Politécnica de Chimborazo. 87 p. 
12. Flores A, Hernández A, Valladares M. 2004. Determinación de la actividad antifúngica de aceites esenciales extraídos de Lippia graveolens (Orégano), Rosmarinus officinalis (Romero) y Eucalyptus globulus (Eucalipto) en Microsporum canis, Trichophytum rubrum y Epidermophyton floccosum. Tesis de Licenciatura. San Salvador, El Salvador: Universidad de El Salvador.96p.

13. Guaguere E. 2007. Terapia dermatológica del perro. $3^{\circ}$ ed. España: Elsevier. $272 \mathrm{p}$.

14. Guerra L. 2011. Evaluación de la actividad antimicrobiana y antioxidante de aceites esenciales de plantas usadas en medicina tradicional. Tesis de Maestría. México: Universidad Autónoma de Nuevo León. $118 \mathrm{p}$.

15. Lizcano A, Vergara J. 2009. Evaluación de la actividad antimicrobiana de los extractos etanólicos y aceites esenciales de las especies vegetales Valeriana pilosa, Hesperomeles ferruginea, Myrcianthes rhopaloides y Passiflora manicata frente a microorganismos patógenos. Tesis de Grado. Bogotá, Colombia: Pontificia Universidad Javeriana. $131 \mathrm{p}$.

16. López M. 2008. El romero, planta aromática con efectos antioxidantes. OFFARM 27: 60-63.

17. Maddison J, Page S, Church D. 2004. Farmacología clínica en pequeños animales. Argentina: Inter-Médica. $300 \mathrm{p}$.

18. Plumb D. 2010. Manual de farmacología veterinaria. $6^{\circ}$ ed. Argentina: Inter-Médica. $1256 \mathrm{p}$.
19. Purca T. 2013. Efectividad antibacteriana «in vitro» del extracto etanólico de Rosmarinus officinalis (romero) sobre la flora salival. Tesis de Cirujano Dentista. Lima: Universidad Nacional Mayor de San Marcos. $97 \mathrm{p}$.

20. Rodríguez D, Kozusny-Andreani D. 2010. Utilização de extratos de plantas medicinais e óleo de Eucaliptus no controle in vitro de Microsporum canis. Rev Cub Plantas Med 15: 119-125.

21. Ruiz M, Nieto D, Larios R. 1975. Tratado elemental de botánica. $13^{\mathrm{a}}$ ed. México: Ed ECLAL. 655 p.

22. Ruiz J, Roque M. 2009. Actividad antimicrobiana de cuatro plantas de nororiente peruano. Ciencia e Investigación 12: 41-47.

23. Scott D, Miller W, Griffin C. 2002. Dermatología en pequeños animales. $2^{\circ} \mathrm{ed}$. Argentina: Inter-Médica. 1349 p.

24. Sumano H, Ocampo L. 1997. Farmacología veterinaria. $2^{\circ}$ ed. México: McGraw-Hill. 1000 p.

25. Tabanca N, Demirci B, Baser K, Aytac Z, Ekici M, Khan S, Jacob MR, Wedge DE. 2006. The chemical composition and antifungal activity of Salvia macrochlamys and Salvia recognita essential oils. J Agric Food Chem 54: 6593-6597.

26. Teixeira L. 2012. Avaliação do uso do extrato de alecrim de jardim (Rosmarinus officinalis Linn) no controle do biofilme dental. Tesis de Cirujano Dentista. Curitiba: Universidad Federal de Paraná. 28 p. 